\title{
AUTOMATIC FAÇADE SEGMENTATION FOR THERMAL RETROFIT
}

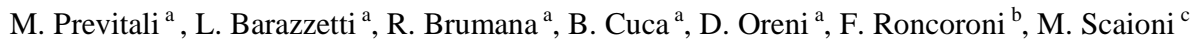 \\ ${ }^{a}$ Politecnico di Milano, Department of Architecture, Built Environment and Construction Engineering \\ Via Ponzio 31, 20133 Milano, Italy - \\ mattia.previtali@mail.polimi.it, (luigi.barazzetti, raffaella.brumana, branka.cuca, daniela.oreni)@polimi.it \\ ${ }^{\mathrm{b}}$ Politecnico di Milano, Polo Territoriale di Lecco, Via Marco D'Oggiono 18/A, Lecco, Italy - \\ fabio.roncoroni@polimi.it \\ ${ }^{c}$ Tongji University, College of Surveying and Geo-Informatics \\ 1239 Siping Road, 200092 Shanghai, P.R. China - \\ marco@tongji.edu.cn
}

KEY WORDS: Façade segmentation, Laser scanning, Texturing, Thermal images

\begin{abstract}
:
In this paper we present an automated method to derive highly detailed 3D vector models of modern building façades from terrestrial laser scanning data. The developed procedure can be divided into two main steps: firstly the main elements constituting the façade are identified by means of a segmentation process, then the 3D vector model is generated including some priors on architectural scenes. The identification of main façade elements is based on random sampling and detection of planar elements including topology information in the process to reduce under- and over-segmentation problems. Finally, the prevalence of straight lines and orthogonal intersections in the vector model generation phase is exploited to set additional constraints to enforce automated modeling. Contemporary a further classification is performed, enriching the data with semantics by means of a classification tree. The main application field for these vector models is the design of external insulation thermal retrofit. In particular, in this paper we present a possible application for energy efficiency evaluation of buildings by mean of Infrared Thermography data overlaid to the façade model.
\end{abstract}

\section{INTRODUCTION}

Due to the increasing capabilities of terrestrial laser scanning (TLS) technology during the acquisition phase, geometric models are rapidly growing in size and complexity. In particular, nowadays an increasing interest is paid to the generation of detailed building models from TLS data (Becker and Haala, 2007). This is due to the fact that the raw point cloud generation process is quite simple and highly automated, reducing this way the time for data acquisition and registration. However, raw point clouds are generally not directly used for practical applications like urban planning, environmental impact assessing, production of executive drawings for retrofit interventions, etc. This is due by the fact that point clouds present a very low level of abstraction. In addition, further problems arise due to the huge size of data to be managed. For these reasons the point cloud is generally vectorized, and possibly enriched with semantic information, allowing in this way an higher-level of interaction to the user. Indeed, operations such as editing, moving and replacing objects in the vectorized model, make the data more usable for modelling purposes. Of course, traditional reverse engineering approaches can be applied. However, the process is generally time consuming because involves a quite tedious user interaction. In addition, highly skilled operators are needed. The automatic reconstruction of building models can be used in conjunction with or as an alternative to computer-aided-design, allowing an increase of efficiency and a cost reduction. In fact, manual editing can be avoided if a detailed reconstruction is not required, or shall take place only after a basic model has been automatically generated. Although much work has been done practical application to automatic modeling of full façades is still far to be achieved. This is mainly due to the wide plethora of architectural elements and styles that could be combined in façades.

The goal of this paper is to present an automatic approach for planar object extraction and 3D model generation starting from point clouds of building façades acquired by TLS. The developed methodology can be applied to scenes of tens of millions input points, in order to produce highly idealized 3D vector models. In the developed methodology the unorganized point cloud is firstly segmented to identify the main façade elements. As in modern buildings the majority of façade components are planar the segmentation is aimed at detecting planar elements, being the detection of other kinds of shapes a future work. Identification of such elements is performed by using a RANSAC implementation. In order to reduce problems connected to bad-segmentation results of RANSAC in the case of massive point clouds, topology information is included in the process. In particular, bad-segmentation results may be categorized into:

$$
\begin{aligned}
& \text { i. under-segmentation, in the case several features } \\
& \text { are segmented as one; } \\
& \text { ii. over-segmentation, one feature is segmented }
\end{aligned}
$$
into several.

Once façade main elements are detected, the vector model is then generated adding some priors on façade geometry. In particular, the prevalence of straight lines and orthogonal intersections is exploited as additional constraint to enforce the modeling process. Contemporary a further recognition and separation of planar object is performed by using a classification tree. Results of this classification are helpful to enrich data with semantics for modeling at Level-Of-Detail 3 (LOD3).

An application of the developed method concerned the energy efficiency evaluation of buildings by mean of Infrared 
Thermography (IRT) data overlaid to the 3D façade model. Indeed, infrared thermography can be effectively used to determine thermal bridges and other thermal anomalies in the building envelop. The relationship between thermal data and building geometry is of primary importance in order to precisely locate surfaces affected by thermal defects. In this paper a fusion of those two input data is obtained by mapping thermal images on the façade model.

\section{RELATED WORK}

In recent years a large number of segmentation method dealing with point cloud has been proposed. Segmentation can be defined as the process aimed at grouping points sharing a spatial similarity. Initially segmentation techniques were developed for airborne laser data (Masaharu and Hasegawa, 2000; Geibel and Stilla, 2000). Some algorithms developed on airborne laser data proved their suitability also to TLS data for extracting planar and façade surfaces (Lerma and Biosca, 2005; Pu and Vosselman, 2006; Stamos et al., 2006).

The developed segmentation methods can be classified into three main groups:

$$
\begin{array}{cl}
\text { i. } & \text { region growing; } \\
\text { ii. } & \text { clustering of features; } \\
\text { iii. } & \text { model fitting. }
\end{array}
$$

Region growing methods start at a seed point and identify homogeneous points, based on certain similarity criteria around the seed. In the case of planar element identification ( $\mathrm{Pu}$ and Vosselman, 2006) a seed with its nearby points is chosen. If a plane fits points within a user-defined threshold the surface is accepted and the growing procedure starts. The growing can be based on: point proximity, global planarity or point normal vector direction. However, several extensions for surface growing methods have been suggested. Generally, region growing methods suffers from the correct identification of a seed point. An initial wrong selection might lead to wrong segmentation results.

The methods based on clustering of features (Filin, 2004) can be seen as a combination of two process: identification of patterns in the data based on some attributes and then data clustering. Segmentation results of this technique depend on the selected features. In addition clustering multidimensional features for large point cloud can be computationally infeasible. Model fitting methods (Schnabel et al., 2007; Boulaassal et al., 2008) try to identify geometrical primitives in the point cloud. Points conforming to a defined shape are grouped as belonging to the same segment. Algorithms based on model fitting mainly relies either on RANSAC (Fischler and Bolles, 1981) or on Hough-transform (Hough, 1962). In particular, RANSAC proved to be more efficient in the case of laser scanner data (Tarsha-Kurdi et al., 2007). However, RANSAC measures only the consensus to a certain assumed model. Consequently, the extracted planes may not correspond to real architectonical object. This is generally the case of intertwined planar objects. For example, in the case two or more different objects (like different windows) are evaluated belonging to the same geometrical plane they are erroneously clustered as a single segment, determining an under-segmentation problem. On the other hand, over-segmentation problems may arise in the case of an out of plumb in the façade. This non planarity may determine the segmentation of the main façade plane into several objects. In this paper, we present an extension of standard RANSAC approach to overcome these bad-segmentation problems. Although the segmentation problem has been widely covered in literature, only in recent years the goal of further parameterized high-level models emerged. Several methods were proposed to process the data with user interaction. An interactive tool for assembling architectural models directly over 3D point clouds acquired from TLS data is introduced in Nan et al. (2010): users define simple building blocks which are then snapped to similar architectural objects. In Zheng et al. (2010) an interactive method for holes completion in scans of building façades and manual modeling is presented. Other interactive approaches aim at fusing TLS data with images for layered and textured polygonal building model generation ( $\mathrm{Li}$ et al, 2011). However, extended processing time and long human modeling is needed. There exist also approaches that aim at full automation. In Pu and Vosselman (2009) an automatic reconstruction method is presented: starting from TLS data segmentation, data are then fitted to generate detailed polygonal façade models. Recently, in (Vanegas et al., 2012) an approach based on Manhattan World building geometry assumption was presented. This system classifies data into features. These features are then grouped in connected clusters and a volumetric model is generated. However, due to the wide variety of architectural elements and styles, practical application to automatic modeling of full facades is still far to be achieved. For a complete review about this topic we address to Musialski et al. (2012).

In this paper, we present an automatic reconstruction methodology incorporating priors on façade geometry. In particular the prevalence, in modern building, of straight lines and orthogonal intersection between adjacent planes.

Some papers addressed the problem of enriching building models with semantics. Most of them focus on exploiting the regularity on façade elements (like windows) to classify façade objects. In Ripperda and Brenner (2006) a reconstruction strategy based on a formal grammar and reversible jump Markov Chain Monte Carlo (rjMCMC) is proposed. An automatic extraction and classification of building features is presented in $\mathrm{Pu}$ and Vosselman (2009), whereas the structure and hierarchy of the façade are described in form of shape grammar in Becker (2009). This paper presents a simple and intuitive approach to automatically recognize façade features. The proposed method is based on knowledge of geometrical and topological configuration of façade objects and is guided by a classification tree.

In addition, the Integration of IRT and TLS data is also addressed. Here, many solutions are focused in defining procedures for a rigorous co-registration of this kind of data. In many applications thermal data registration is carried out by using homographic transformations, one per each image to map (González-Jorge et al., 2012). Some specific acquisition systems were designed to speed up the thermal image orientation. A "bicamera" system, coupling a traditional RGB camera with a IR sensor, can be used. In this case only RGB images are processed in the bundle adjustment deriving their exterior orientation. Then, starting from the relative orientation between RGB and IR cameras determined in a calibration phase, thermal images are oriented (Alba et al., 2011). In the case of mobile mapping systems integrating IR cameras the image orientation can be performed in a two-step way. Thermal images are firstly relatively oriented using the Neister 5-point algorithm. Then, the obtained sequence is matched to the recorded GPS camera path and then to the building façade (Hoegner and Stilla, 2009). The method here presented differs from the most existing approaches because it relies on a rigorous photogrammetric orientation of both thermal and RGB images computed together in a combined bundle adjustment. 


\section{THE METHODOLOGY FOR FAÇADE MODELING}

The developed approach for façade modeling allows for automatic planar object extraction and 3D vector model generation starting from point clouds of building façades acquired with TLS. To achieve this task, the input data is a raw unstructured point cloud (Figure 1), which can be produced by using a single or multiple station acquisition. Indeed, after scan registration/geo-referencing, multiple scans are merged together without needing any reorganization into a specific data structure.

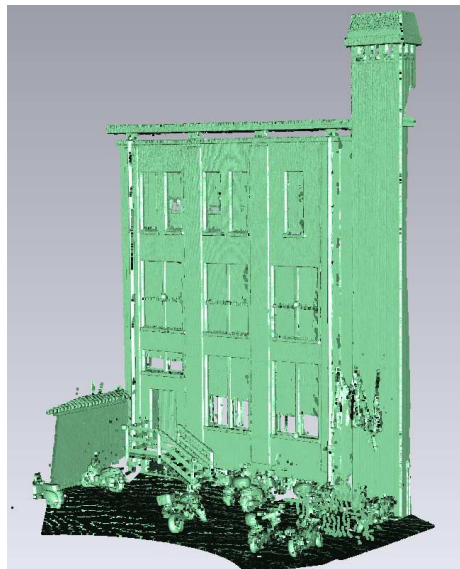

Figure 1. Raw point cloud of a building façade (about 15 million points).

A first remark is needed, several algorithms presented in this work rely on normal vector information at the point locations. However, scanning devices usually do not provide measurements of surface normal. Therefore, point normal usually need to be estimated. Point normal estimation can be performed using the approach proposed by Jenke et al. (2008): for each point in the cloud the 10-nearest neighbors are considered and a plane is fitted. Afterward, the number of nearest neighbors is increased iteratively until eigenvalues of the covariance matrix and point normal stabilize.

Once normals are added to original points the proposed methodology can be divided in three steps: (i) point cloud segmentation, (ii) vector model generation, (iii) object classification.

\subsection{Point cloud segmentation}

As a first task the unorganized point cloud is segmented to identify the different façade main elements. Since the majority of facade components are planar (for the building analyzed in this paper) the segmentation is aimed at detecting planar elements, being the detection of other more complex shapes the object future work. Planar objects are extracted by using a modified RANSAC algorithm (Figure 2). In each iteration of the algorithm the plane with maximal score is searched using the RANSAC principle, once the maximal consensus plane is detected, the corresponding inliers are removed from the point cloud. The algorithm terminates as soon as the detected planar object is lower than an user defined minimal size. However, many papers showed two major shortcomings of RANSAC in the case of massive point cloud segmentation:

i. RANSAC relative inefficiency in terms of computational time, ii. generation of spurious planes produced with points extracted from different objects in the point cloud

In this paper less care is paid to RANSAC computational inefficiency. However, the adaptive variant proposed by (Hartley and Ziesserman, 2003) was implemented in order to determine adaptively the number of samples to be tested, reducing in this way the processing time (if compared to the basic approach).

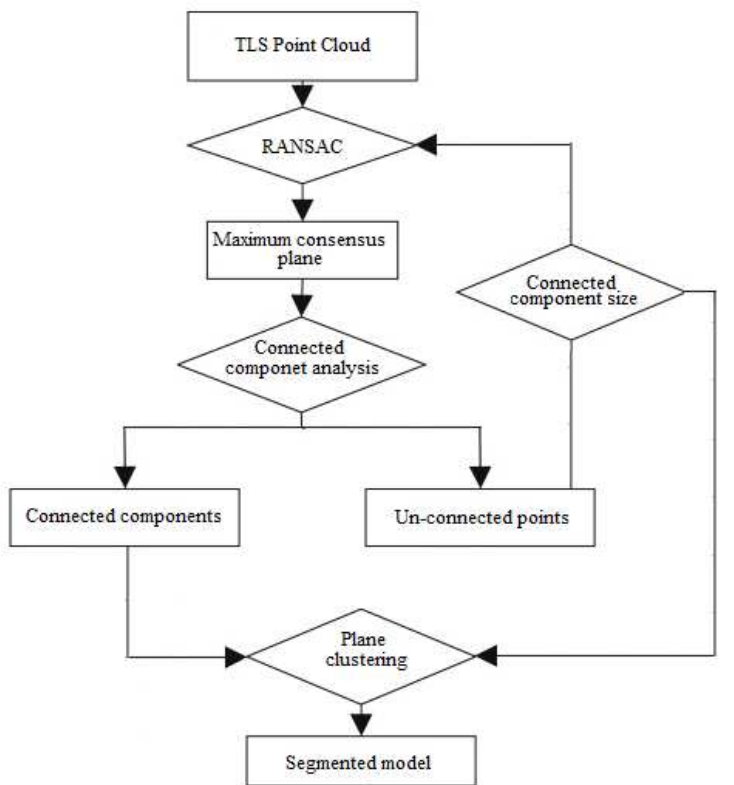

Figure 2. Segmentation process.

A major attention was paid to the reduction of badsegmentation problems. As previously mentioned spurious results may be due to the fact that points constituting the maximum consensus to RANSAC planes are derived from different objects. The first strategy to prevent this problem is to check the consistency of point normal vectors and the estimated RANSAC plane normal. Indeed, once three points are sampled from the cloud and the plane is estimated, inliers evaluation take into consideration not only the distance between each point and the plane but also the angular distance between each point normal vector and the estimated plane normal. In this way we prevent points belonging to planes having different orientations being clustered together. However, this strategy does not consider if different object belonging to the same plane are clustered in a single feature. This is the typical case of windows (Figure 3). The methodology used for modifying RANSAC to avoid under-segmentation problem is the introduction of topology information between points. Indeed, points belonging to the same object should be sufficiently close while groups of points belonging to different object should have a gap area. For this reason, a topology measure is introduced in the segmentation process by identifying objects supported by a connected component point dataset. Once a maximum consensus plane is defined the connectivity between points is measured by analyzing a bitmap generated from the points belonging to the RANSAC plane. The bitmap is obtained by projecting each points orthogonally along the normal direction, if at least one point is projected into a pixel its value is set to 1 otherwise the pixel value remains equal to 0 (Figure $3 \mathrm{a}$ ). The pixel size in the bitmap is chosen as the mean sampling resolution in the point cloud. Once the bitmap is setup the connected components can be easily defined, then all points whose projection belongs to the same connected component are 
grouped in the same segment (Figure 3b). In this phase some spurious segments can be individuated and rejected. Indeed, each segment whose area is lower than a user defined minimal size are rejected and corresponding points are not removed from the point cloud.

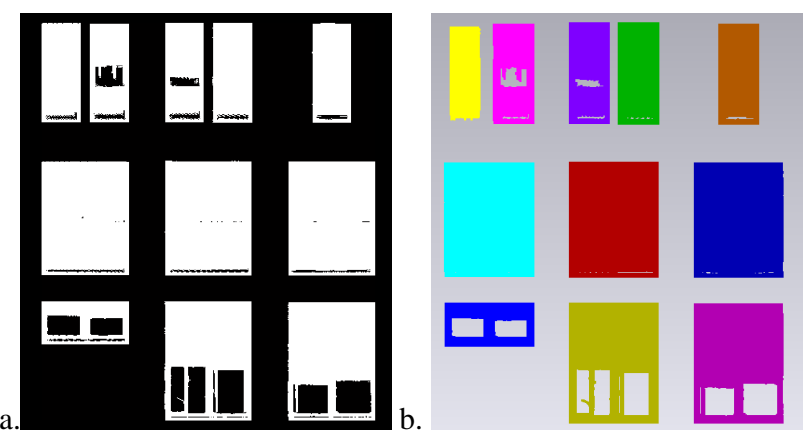

Figure 3. Segmentation of façade window: generated bitmap (a), connected component detected (b).

In the segmentation process over-segmentation problems may also arise. In this case a single object is segmented into several clusters. This is generally due to noise into the data, bad scan registration or imperfections in the construction. Indeed, façades may present some out of plumb, whose size may not be relevant for the desired Level-of-Detail. In those cases the façade plane is segmented into two or more intertwined clusters while the expected result is a unique feature (Figure 4). Oversegmentation may be prevented increasing the RANSAC tolerances. However, this may lead to under-segmented parts which may be difficult to split again. For this reason, in our work a moderate over segmentation is preferred during plane detection phase. Then, once all objects are detected, oversegmented parts are combined together considering topology properties of extracted planes. In particular, three parameters are evaluated: (i) similarity of normal vectors, (ii) perpendicular distance between planes, (iii) intersection between planes. The developed methodology firstly clusters the extracted planes according to normal vector by using the mean shift algorithm (Comaniciu, 2002).

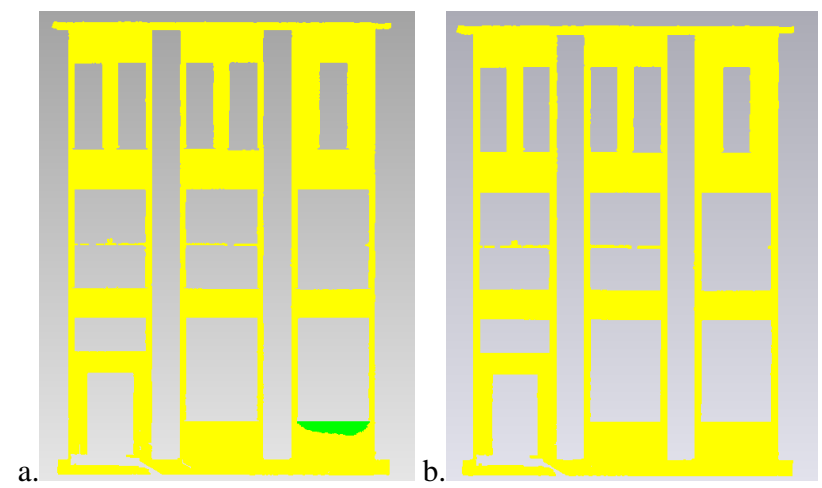

Figure 4. Façade over-segmentation: (a) a small out of plumb (1 $\mathrm{cm}$ ) is detected in the façade (green cluster), (b) clusters are merged after clustering all planes.

Then, a new grouping is performed into each cluster. The mean distance between a plane and points clustered into another RANSAC detected plane is considered. If this distance is inferior to the user defined threshold and the convex hulls of the two clusters intersects they are recognized as a single plane and fused together into a unique object.

\subsection{Vector model generation}

Once planar clusters constituting the façade are determined by the developed segmentation approach the first step for a further parameterized high-level vector model generation is the extraction of cluster contours. For this purpose a contour extraction algorithm enforcing straight line prior has been developed. However, as a preliminary step a new coordinate system defined by the planar cluster is determined performing a Principal Component Analysis (PCA) on the points of the cluster (the two orthogonal planar basis and the normal vector are determined). In this new principal component space the new coordinates of points are calculated from the original ones $\left(\mathrm{X}_{\text {or. }}\right.$. $\mathrm{Y}_{\text {or. }}, \mathrm{Z}_{\text {or. }}$ ). In particular, $\mathrm{Z}_{\text {new }}$ may be considered as negligible. Once the new coordinates are calculated $\left(X_{\text {new }}, Y_{\text {new }}\right)$ a binary image (Fig. 5a) for the cluster can be generated. In this image white pixels represent elements where TLS data are available, while black pixels are grid elements with no data (Fig. 5b). The optimal pixel size, as previously discussed, is a the mean sampling resolution in the point cloud. In this binary images cells representing an edge can be easily identified. Of course, accuracy of extracted edges is influenced by pixel size and in case of a coarse rasterization the final accuracy can be insufficient.
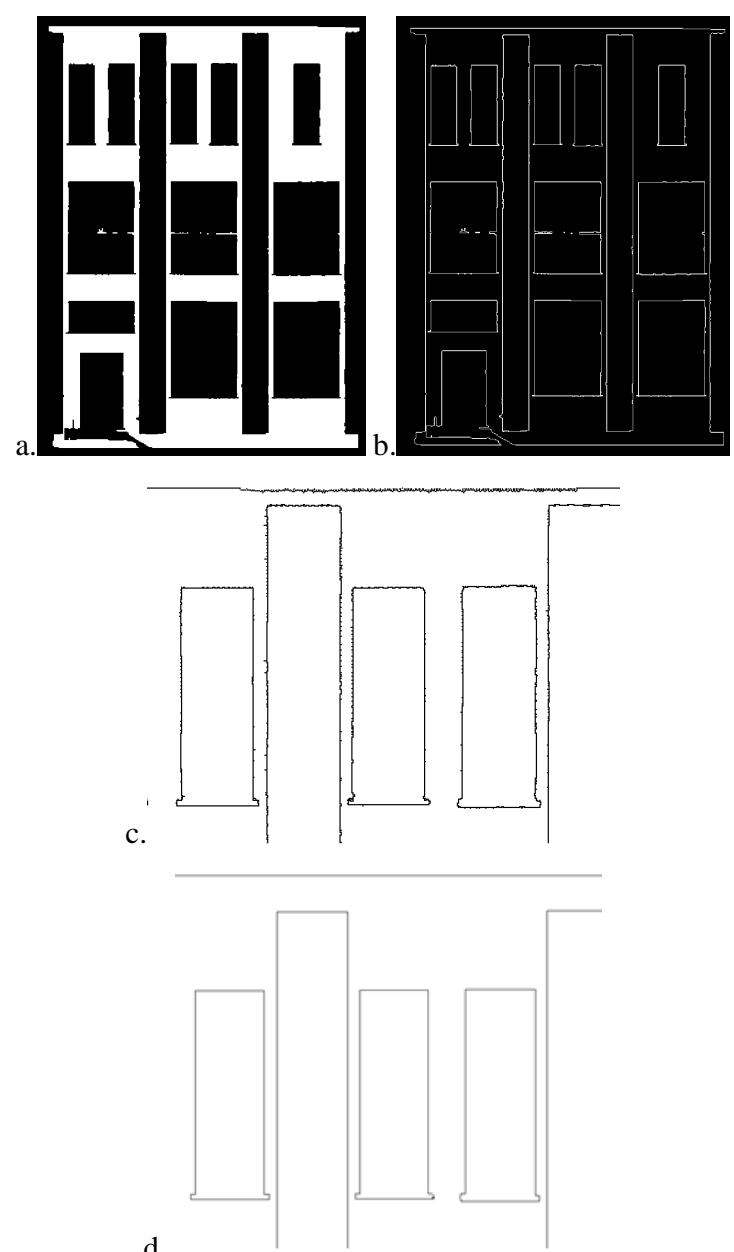

Figure 5. Edge extraction: (a) the binary image, (b) edges from the binary image, boundary edges before (c) and after (d) the straight line constraint.

However, this limited accuracy is acceptable since the binary image is used only to classify cells into edge and non-edge cells, while the contours are computed by using the original points 
belonging to edge cells. In particular, a Delaunay triangulation is performed considering all points belonging to those cells. As observed in (Boulaassal, 2007) contour points can be individuated as extremities of long sides of Delaunay triangles. However, contours obtained in this way are quite irregular showing a sawtooth shape (Fig. 5c). On the other hand, in façade elements straight lines are predominant, in addition lines are mainly horizontal or vertical. For this reason, once the contour points are determined the dominant edge directions are identified by using a RANSAC implementation aimed at detecting dominant lines. In particular, in the case nearly horizontal or vertical directions are detected horizontal or vertical constraints are forced in the line generation phase (Fig. $5 d)$. Once the edges are extracted they are transposed in the original datum.

Finally, the connection between surfaces is verified. Indeed, after the use of the RANSAC algorithm the connection between surfaces is generally lost. This is due to the fact that in the case of intersecting surfaces the points on the intersection are assigned only to one cluster. In order to enforce surface intersection nearly intersecting objects are firstly identified verifying the distance between detected edges. If this distance is lower than the RANSAC threshold used for plane detection the planes are designed as nearly intersecting.

Once two nearly intersecting surfaces are identified their intersection is enforced. In this way, not only topology between object is re-establisher but also accuracy in edge detection is increased (Fig. 6). Indeed, the edge is calculated as intersection of planes estimated from a large set of points. While edge calculation from contour points may be more affected by noise in the point cloud. In addition, accuracy of laser scanning measurements in correspondence of edges is generally lower than the one on smooth surfaces.

(a)

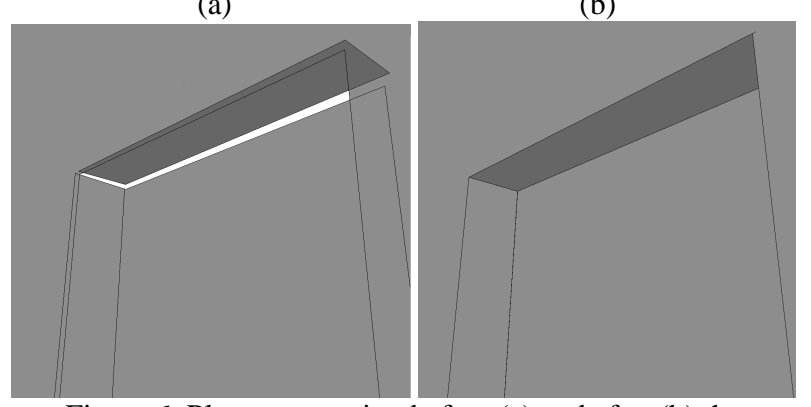

Figure 6. Plane connection before (a) and after (b) the intersection constraint.

Once straight line and surface intersection constraints are applied the vector model can be exported into a CAD environment for manual editing and drawing production. For this reason, the model is exported into a .DXF (Drawing eXchange Format) file containing the extracted edges. However, in many application surface models are needed and a constrained 2D Delaunay triangulation is used to achieve this task. For each detected planar primitive the reconstructed object boundary are used as constraints (Fig.7).
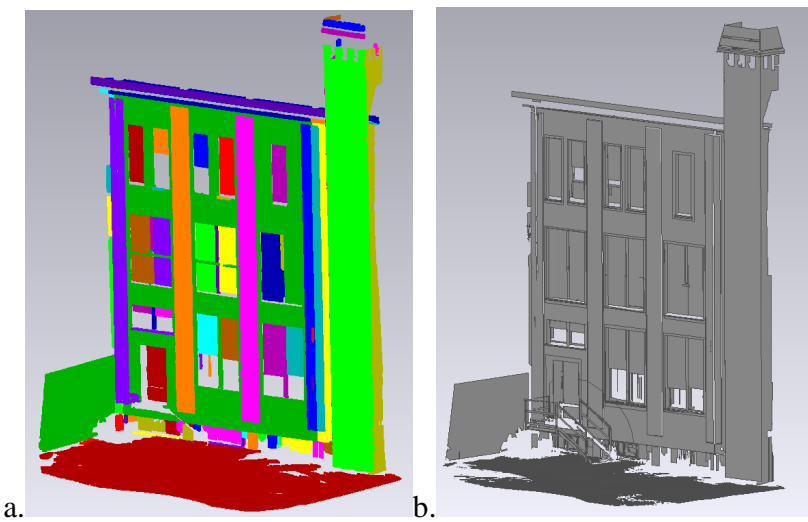

Figure 7. Detected planar elements (a) and polygonal mesh (b).

\subsection{Object classification}

The segmentation and the vector model generation phases lack in determining which kind of semantic feature a detected object represents. To enrich the facade model with semantics a further classification can be accomplished by observing that each semantic feature has some peculiar characteristics which can be formulated as recognition rules. These rules can be derived either from statistical analysis of train sets or they can based on façade and urban scene geometry priors. The former has the main disadvantage of an out-of-core training phase based on a wide number of manually labelled cases. However, due to statistical analysis they can be used for a wide variety of building types. In the second case interpretation rules should be a compromise between two opposing requirements: these rules should be relatively flexible in order to allow their application for different building types, on the other hand rules should allow a clear distinction between different features.

In this paper we present a classification based on recognition rules derived from knowledge of urban scenes and façade priors. In particular, the following characteristics were found to be an efficient trade-offs between the previously defined opposite requirements:

i. object size: walls can be easily distinguished by non-wall elements because they have a larger area.

ii. object position and topology: for example, we assume that doors can be only at the bottom floor, roofs are always on the top of walls and the ground is the object at the lowest level.

iii. Object orientation: walls are assumed vertical, while ground and roofs are generally horizontal or mildly sloped.

The above object characteristics and priors are summarized in a classification tree (Figure 8) which is used to guide the classification phase. The six semantics features extracted are wall, window, door, roof, ground and wall attachments. In particular, the classification begin evaluating both area and position of all the detected objects. First, the ground is detected because it is the horizontal (or pseudo-horizontal) object at the lower level. Then the main façade planes are extracted since they are objects perpendicular to the ground and have the largest area with respect to all other objects. Indeed, the wall area in a façade is generally much larger than the one covered by other objects like windows or doors. In addition, main façade planes are generally less than sidewalls, windows and doors, so they can be considered as outliers and can be easily detected by robust statistics. 


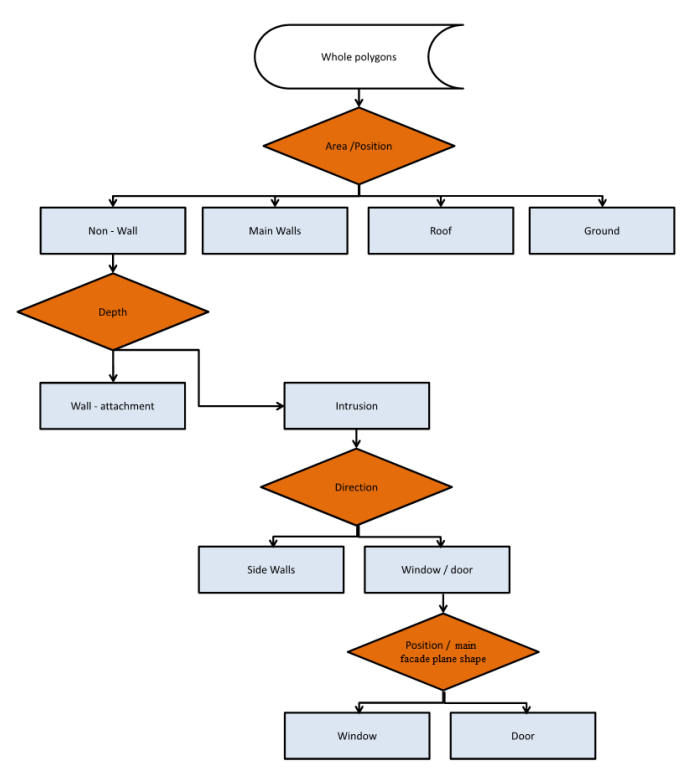

Figure 8. Classification tree, orange diamonds are conditions while blue rectangles represent facade elements.

Then flat-like objects over walls protruding out of the façade are classified as roof. For all non-classified objects their position with respect to the main façade plane is evaluated. Objects in front of the façade are extrusion objects and classified in a general way as wall attachments while other ones are classified as intrusions. Intrusions can be distinguished in sidewalls, windows and doors. Sidewalls differ from other intrusions because their orientation is perpendicular to the frontal face of the walls and for this reason they can be easily recognized. In order to distinguish between doors and windows it is assumed that doors are only at bottom floor. In addition in correspondence of a door the main façade plan has a characteristic gap having an inverted "U shape" while in correspondence of windows the gap has an "O shape". Starting from this considerations doors are searched only at the ground floor and in correspondence of inverted $U$ shape gaps in the main wall face. Other intrusions parallel to the façade plane are classified as windows. The derived semantics information are used to generate a semantically rich CityGML 3D model at LOD3 (Fig.9).

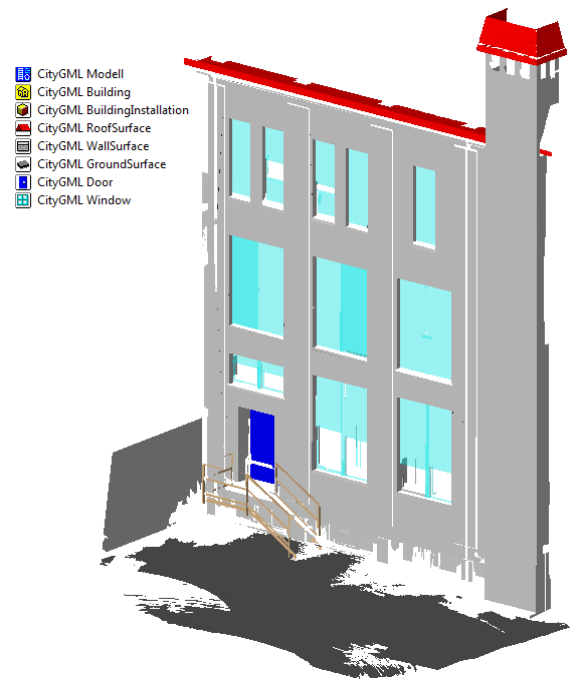

Figure 9. The CityGML façade model.
CityGML is the international standard for the representation and exchange of $3 \mathrm{D}$ city models. The main advantage of this markup language is the fact that it defines not only the threedimensional geometry of an object but also topology and semantics. This is in contrast to purely geometrical/graphical models such as KML or VRML, which do not provide sufficient semantics. All geometry and semantics definitions are provided in different, well-defined Levels-of-Detail (multiresolution model). For a complete overview about CityGML we address to Gröger and Plümer (2012).

\section{TEXTURE MAPPING OF 3D MODELS WITH THERMAL IMAGES}

The main application field for the derived models is the design of proper solution for external insulation thermal retrofit. In order to plan an intervention a detailed geometrical model of the building is necessary for a precise location of thermal bridges and thermal anomalies, and for the production of executive drawings. In this paper we present an application of the developed reconstructing procedure for the energy efficiency evaluation of buildings by mean of Infrared Thermography (IRT) data coupled with the automatically derived façade model. Indeed, the relationship between thermographic data and the building geometry is of primary importance in order to precisely locate surfaces affected by thermal defects. Fusion of those two data sets is performed by mapping thermal images on the $3 \mathrm{D}$ model. In order to achieve accurate data fusion the following steps are required:
i. geometric thermal camera intrinsic calibration;
ii. co-registration of images and $3 \mathrm{D}$ model;
iii. texture mapping of thermal images.

\subsection{Geometric thermal camera intrinsic calibration}

Generally, thermal cameras can be modeled just as a central projection with lens distortion and can be calibrated by using standard photogrammetric methods. However, calibration of IRT cameras may not be an easy task for a series of reason:
i. large lens distortions,
ii. low resolution of IRT sensors,
iii. limited field of view.

For all these reasons a proper planning for the calibration project is needed.

In our application a FLIR Tau 640 with geometric resolution of $640 \times 480$ pixel was calibrated by using the Brown model (Brown, 1971). Geometric camera intrinsic calibration was performed recording a set of 18 images of a calibration polygon materialized with a set of 40 iron nails fixed in a wooden structure (Fig.10a).
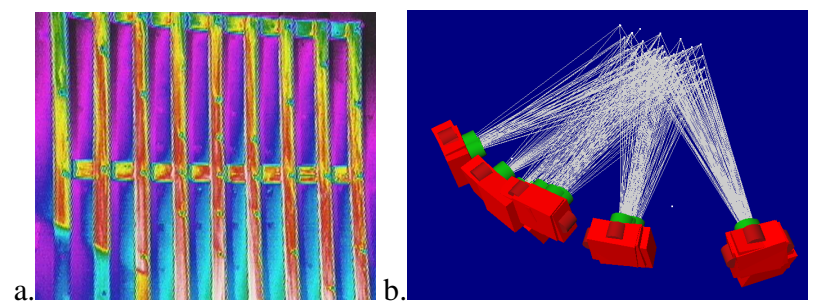

Figure 10. The polygon used for IRT camera calibration (a), IR image arrangement for the camera calibration (b).

Some aspects of the calibration procedure need a special remark. Generally, distortion parameters are generally highly correlated. In particular, in the presented case, some distortion 
parameters showed a correlation larger than $90 \%$ and a precision in the same order of magnitude of the estimated parameter. For this reason, rolled images were introduced in the block to reduce correlations and in the final estimation parameter $\mathrm{k}_{3}$ was forced to zero and only seven parameters were used. Fig. 10b show the difficulty in acquiring strongly convergent images due to the narrow field of view of IR camera.

\subsection{Co-registration of images and 3D model}

The main issue for an accurate texture-mapping is the registration of façade geometric model and thermal images in the same reference system. This task is obtained by using a rigorous photogrammetric orientation of both thermal and RGB images in a combined bundle adjustment (Scaioni et al., 2012). This solution allows one to have a better control on the quality of results with respect to other traditional approaches mainly based on homography or space resection.

The procedure starts with the acquisition of a set of RGB images with a calibrated camera. Then, RGB images are oriented within a standard photogrammetric bundle adjustment, which is based on a set of Tie Points (TPs) measured on the images, and some Ground Control Points (GCPs) that are used to register the project in the reference system of the laser scans. An important consideration deserves to be mentioned: TPs individuated in this first step will be then adopted for registration of IRT images. This means that TPs should be preferably measured in correspondence of elements that are clearly visible in both RGB and IR images (e.g., windows corners). Once RGB images have been registered, IR images can be added to the block. A final bundle adjustment including all data is then carried out to obtain the Exterior Orientation (EO) parameters of all images. This combined adjustment has several advantages:

i. global and local redundancies allow a better estimation of EO parameters better than those estimable with standard space resection techniques;

ii. RGB and IR image acquisition may be carried out independently, even in different days;

iii. this method allows the user to include new data taken at different epochs.
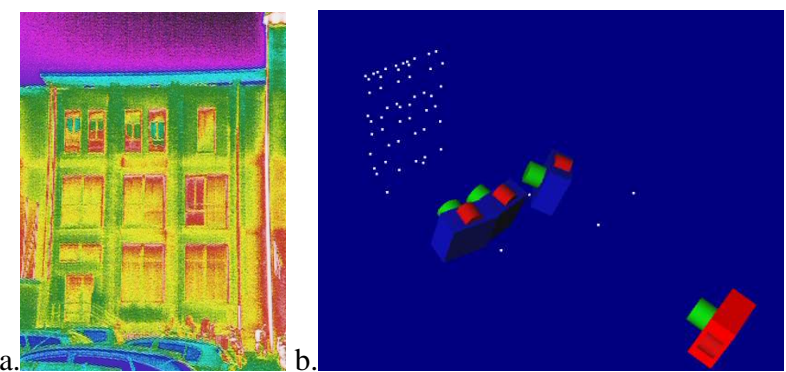

Figure 11. The IR image (a), a 3D view showing camera poses of both RGB (blue) and thermal (red) cameras (b).

In this case 3 RGB images acquired with a Nikon D700 with a $35 \mathrm{~mm}$ lens were acquired in conjunction with the IR image (Fig.11a). 14 checkerboard targets, measured with a theodolite Leica TS30 were employed for the registration of laser scans and were also used as GCPs during bundle adjustment for RGB image only. In addition 10 tie points in correspondence of window and door corners were measured on both RGB and thermal images to obtain the EO parameters of all images (Fig.11b). Statistics of the combined bundle adjustment show a final sigma-naught of about 0.9 pixels.

\subsection{Geometric thermal camera intrinsic calibration}

Finally, when both data sets (thermal images and 3D façade model) are registered together their fusion is performed by means of texture mapping. Some commercial software packages accomplish texture mapping. However, in many cases the process is manual, meaning that the measurement of correspond points (images $\leftrightarrow$ model) is carried out interactively.

The algorithm here adopted for texture mapping was implemented in order to minimize self-occlusions and texture assignment problems (Previtali et al., 2012). Once texture is applied to the façade model several products can be obtained: 3D triangulated textured models (Fig.12), which allow the visualization of thermal data overlaid to $3 \mathrm{D}$ spatial information or raster datasets like orthophotos, having the temperature as radiometric value, which can be used to evaluate thermal gradients and thermal bridges on the façade surface.

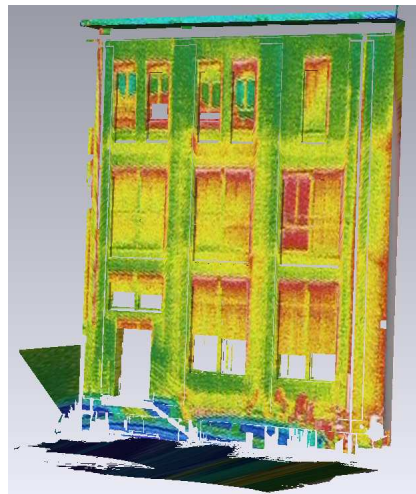

Figure 12. The IR-textured 3D model.

\section{CONCLUSION AND FUTURE WORK}

This paper presented a novel method to derive 3D vector model of building façades from massive unstructured point clouds in an automatic way. The final model proved its usefulness in case of thermal retrofit of the building. Indeed, the vector model can be used both for production of executive drawings of retrofitting intervention and energy efficiency evaluation.

In particular an extension of the RANSAC approach to point cloud segmentation is presented. The developed strategy tries to avoid bad-segmentation results reported in various papers by adding some topological information in the segmentation process. Starting from segmentation results vectorialization of detected objects is automatically performed by introducing verticality, horizontality and orthogonality priors which feature the architectural style of many modern buildings. A semantic enrichment of the model is obtained by using a classification tree to recognize façade objects and allowing modelling building at LOD3. Occlusions and data lacks determine some gaps in the automatically generated model. For this reason the derived models can be used as an alternative to manual CAD models (in the case a simplified modeling is sufficient) or in conjunction with manual model (if a very accurate model is needed). In the second case manual modeling can take place on the automatically generated model allowing an increment of efficiency along with a cost reduction. Finally an application for energy efficiency evaluation is presented by fusing IRT data and the 3D façade model by means of texture mapping. Registration of both sets of data is obtained by using a rigorous bundle adjustment of RGB and thermal images together. 
Future work will focus the regularity of façade elements, especially windows and doors, for a completion of areas affected by severe occlusions and a classification of all the extracted objects.

\section{REFERENCES}

Alba M., Barazzetti L., Rosina E., Scaioni M. and Previtali M., 2011, Mapping infrared data on terrestrial laser scanning 3D models of buildings", Remote Sensing, 3(9), pp. 1847-1870.

Becker S. and Haala N., 2007, Refinement of Building Facades by Integrated Processing of LIDAR and Image Data. In PIA07.International Archives of Photogrammetry, Remote Sensing and Spatial Information Sciences (Sept. 2007).

Becker, S., 2009. Generation and application of rules for quality dependent facade reconstruction. ISPRS Journal of Photogrammetry and Remote Sensing, 64(6), pp. 640-653.

Boulaassal H, Landes T and Grussenmeyer P, 2008, Automatic extraction of planar clusters and their contours on building façades recorded by terrestrial laser scanner. VSMM 2008 - Conference on Virtual Systems and MultiMedia Dedicated to Digital Heritage. Limassol Cyprus - October 20th - 25th.

Brown D., 1971, Close-range camera calibration, Photogrammetric Engineering and Remote Sensing, 37(8), pp 855-866.

Comaniciu D. and Meer P., 2002, Mean shift: A robust approach toward feature space analysis. IEEE Trans. Pattern Anal. Machine Intell., 24:603-619.

Filin S., 2004, Surface classification from airborne laser scanning data, Computers and Geosciences 30 (9-10) (2004) 1033-1041.

Fishler M.A. and Bolles R.C., 1981, Random sample consensus: a paradigm for model fitting with application to image analysis and automated cartography. Communications of the AMC, 24(6) : 381-395.

Geibel R. and Stilla U., 2000, Segmentation od laser altimeter data for building reconstruction: different procedures and comparison, Proc. Internalional Archives of Photogrammetry and Remote Sensing, Amsterdam, Vol. XXXIII, Part B3, pp.326-334.

González-Jorge H., Lagüela S., Krelling P, Armesto J. and MartínezSánchez J., 2012, Single image rectification of thermal images for geometric studies in façade inspections, Infrared Phys Technol 55(5):421-6. (2012).

Gröger G. and Plümer L., 2012, CityGML-Interoperable semantic 3D city models, ISPRS Journal of Photogrammetry and Remote Sensing, 71, 12-33.

Hartley R. and Ziesserman A., 2003, Multiple View Geometry in Computer Vision. Second edition. Cambridge University Press, Cambridge. 655 pages: $117-121$.

Hoegner L. and Stilla U., 2009, Thermal leakage detection on building façades using infrared textures generated by mobile mapping, Urban remote sensing event, 2009 jointIEEE.

Hough P.V.C., 1962, Method and Means for recognizing complex patterns. U.S. Patent 3,069,654.

Jenke P., Krückeberg B., and Strasser W., 2008, Surface reconstruction from fitted shape primitives. In 13th International FallWorkshop Vision, Modeling and Visualization, pages 31-40.

Lerma J.L. and Biosca J.M., 2005, Segmentation and filtering of laser scanner data for cultural heritage. CIPA 2005 XX International Symposium, 26 September - 01 October, 2005, Torino, Italy.
Li Y., Zheng Q., Sharf A., Cohen-OR D., Chen B. and Mitra N.J., 2011, 2D-3D fusion for layer decomposition of urban facades. In 2011 International Conference on Computer Vision (Nov. 2011), IEEE, pp. 882-889. 11.

Masaharu H. and Hasegawa H., 2000, Three-dimensional city modelling from laser scanner data by extractiong building polygons using region segmentation method, Proc. Internalional Archives of Photogrammetry and Remote Sensing, Amsterdam.

Musialski P, Wonka P., Aliaga D.G., M. Wimmer, van Gool L. and Purgathofer W., 2012, A survey of urban reconstruction. In EUROGRAPHICS 2012 State of the Art Reports.

Nan L., Sharf A., Zhang H., Cohen- OR D. and Chen B., 2010, SmartBoxes for interactive urban reconstruction. ACMTransactions on Graphics 29, 4 (July 2010), 1.17,19.

Previtali M., Barazzetti L. and Scaioni M., 2012, An automated and accurate procedure for texture mapping from images, Proc. 18th IEEE Intern. Conference on Virtual Systems and MultiMedia (VSMM), G. Guidi / L. Addison (Eds.), pp. 591-594. Milan, Italy, (2012).

$\mathrm{Pu}$ S. and Vosselman G., 2006, Automatic extraction of building features from terrestrial laser scanning. IAPRS, vol. 36, part 5, Dresden, Germany, September 25-27, 5 p. (on CD-ROM).

$\mathrm{Pu}$ S., Vosselman G., 2009, Knowledge based reconstruction of building models from terrestrial laser scanning data. ISPRS Journal of Photogrammetry and Remote Sensing 64, 6 (Nov. 2009), 575-584.

Ripperda N. and Brenner C., 2006, Reconstruction of facade structures using a formal grammar and rjMCMC. Pattern Recognition, Ed. by Franke, K., et al., Springer, pp. 750-759

Scaioni M., Rosina E., Barazzetti L., Previtali M., Redaelli V., 2012, High-resolution texturing of building facades with thermal images. Proc. of SPIE Defence, Security and Sensing, Vol. 8354, 23-27 April, Baltimore, USA.

Schnabel R., Wahl R., Klein R., 2007, Efficient RANSAC for PointCloud Shape Detection. In Computer Graphics Forum,Vol. 26, No. 2, Blackwell Publishing, June 2007, pages 214-226.

Stamos I., Yu G., Wolberg G., Zokai S., 2006, 3D Modeling Using Planar Segments and Mesh Elements. 3rd International Symposium on 3 D Data Processing, Visualization \& Transmission, University of North Carolina, Chapel Hill, June 14-16 2006.

Tarsha-Kurdi F., Landes T. and Grussenmeyer P., 2008, Extended RANSAC algorithm for automatic detectionof building roof planes from Lidar data. The Photogrammetric Journal of Finland, 21(1): 97109.

Vanegas C. A., Aliaga D. G. and Benes B., 2012, Automatic Extraction of Manhattan-World Building Masses from 3D Laser Range Scans. IEEE transactions on visualization and computer graphics, 99.

Zheng Q., Sharf A., Wan G., Li Y., Mitra N. J., Cohen-OR D. and Chen B., 2010, Non-local scan consolidation for 3D urban scenes. ACM Transactions on Graphics 29, 4 (July 2010), 1. 11. 